\title{
A class of Hilfer fractional stochastic differential equations and optimal controls
}

Jingyun LV $\mathrm{LV}^{1}$ and Xiaoyuan Yang ${ }^{1 *}$

"Correspondence: bhxyyang@126.com

${ }^{1}$ LMIB and School of Mathematics and Systems Science, Beihang

University, Beijing, P.R. China

\begin{abstract}
In this paper, we investigate a class of Hilfer fractional stochastic differential equations with nonlocal conditions. We first study the existence of mild solutions of these equations by means of stochastic analysis theory, fractional calculations, and operator semigroup theory. Further, the existence of optimal pairs for the corresponding Lagrange control systems is investigated. Finally, an example is presented to illustrate our obtained results.
\end{abstract}

MSC: $26 \mathrm{~A} 33 ; 60 \mathrm{H} 10 ; 34 \mathrm{A08} ; 93 \mathrm{E} 20$

Keywords: Optimal control; Hilfer fractional derivative; Fractional stochastic differential equations

\section{Introduction}

In the last decades, fractional calculus has attracted considerable attention. It has been widely applied in many areas such as fluid dynamics, thermodynamics, and viscoelastic theory $[1,2]$. The nonlocal property of fractional derivative makes fractional calculus being used in such areas and better results have been obtained. That is, the next state of a system depends not only on its current state but also on all of its historical states. Note that the theory of fractional differential equations (FDEs) is one of the important branches of fractional calculus. In recent years, FDEs in infinite dimensional spaces have been studied extensively since they are abstract formulations for many problems arising from economics, mechanics, and physics. Many researchers focused on the existence of mild solutions of FDEs. In [3], Zhou et al. studied the existence of mild solutions for FDEs with Caputo fractional derivative. By applying the Laplace transform and probability density function, they gave a suitable definition of mild solution. Using the same method, Zhou et al. [4] gave a definition of mild solution for FDEs with Riemann-Liouville fractional derivative. On the other hand, Hilfer [5] proposed a generalized Riemann-Liouville fractional derivative, Hilfer fractional derivative, which includes Riemann-Liouville fractional derivative and Caputo fractional derivative. Hilfer fractional derivative is performed, for example, in the theoretical simulation of dielectric relaxation in glass forming materials. Inspired by [3, 4], Gu et al. [6] gave a suitable definition of mild solution for FDEs with Hilfer fractional derivative. Many authors subsequently studied the Hilfer FDEs in infinite dimensional spaces. For more details on the existence of mild solutions for FDEs, see [7-14] and the references therein.

(c) The Author(s) 2019. This article is distributed under the terms of the Creative Commons Attribution 4.0 International License (http://creativecommons.org/licenses/by/4.0/), which permits unrestricted use, distribution, and reproduction in any medium, provided you give appropriate credit to the original author(s) and the source, provide a link to the Creative Commons license, and indicate if changes were made. 
The optimal control is one of the important concepts in control theory and plays a vital role in control systems. For an optimal control problem, the minimization of a criterion function of the states and control inputs of the system over a set of admissible control functions are necessary. The system is subject to constrained dynamics and control variables, among which additional constraints such as final time constraints can be considered. The optimal control theory has been successfully applied in biology, engineering, economy, physics, etc. (see [15]). In recent years, many efforts have been made to investigated the existence of optimal controls for various types of nonlinear FDEs in infinite dimensional spaces. Wang and Zhou [16] considered the existence of mild solutions for a class of FDEs and optimal controls in the $\alpha$-norm. Guo [17] obtained a second order necessary optimality condition for a class of fractional optimal control problems. Kumar [18] established sufficient conditions for fractional optimal control of system with fixed delay. Zhu [19] studied optimal controls for Riemann-Liouville FDEs without Lipschitz assumption.

On the other hand, the deterministic models often fluctuate due to noise or stochastic perturbation, so it is reasonable and practical to import the stochastic effects into the investigation of FDEs. Meanwhile, the existence of mild solutions and optimal controls for fractional stochastic differential equations (FSDEs) have received great interest of researchers. More precisely, Wang [20] investigated the mild solutions of a class of FSDEs. By constructing Picard type approximate sequences, $\mathrm{Li}$ [21] studied the existence and uniqueness of mild solutions for a class of FSDEs with delay driven by fractional Brownian motion. Ahmed et al. [22] established the existence of mild solutions of Hilfer FSDEs with nonlocal conditions. Yan [23] studied optimal control problems for a class of FSDEs of order $\alpha \in(1,2]$. Balasubramaniam [24] dealt with the solvability and optimal controls for impulsive FSDEs via resolvent operators. Rihan et al. [25] studied the existence of solutions and optimal control of FSDEs with Hilfer fractional derivative and Poisson jumps. For more details, see [26-31] and the references therein.

Motivated by the above discussion, in this paper we study the Hilfer FSDEs with nonlocal conditions in the following form:

$$
\left\{\begin{array}{l}
D_{0^{+}}^{v, \mu} x(t)=A x(t)+f(t, x(t))+\sigma(t) \frac{d W(t)}{d t}, \quad t \in J^{\prime}:=(0, b], \\
\left.I_{0^{+}}^{(1-v)(1-\mu)} x(t)\right|_{t=0}-g(x)=x_{0},
\end{array}\right.
$$

where $D_{0^{+}}^{v, \mu}$ denotes the Hilfer fractional derivative, $v \in[0,1], \mu \in\left(\frac{1}{2}, 1\right)$. Let $J=[0, b] . A$ is the infinitesimal generator of a strongly continuous semigroup $\{S(t)\}_{t \geq 0}$ in a real separable Hilbert space $X$. Let $(\Omega, \mathscr{F}, P)$ be a complete probability space equipped with a filtration $\left\{\mathscr{F}_{t}\right\}_{t \in[0, b]}$ that satisfies the usual hypotheses. The state $x(\cdot)$ takes values in $X$. Let $K$ be another separable Hilbert space. $W: J \times \Omega \rightarrow K$ is a standard Q-Wiener process. $f: J \times X \rightarrow X, \sigma: J \rightarrow L_{2}^{0}$, and $g: \mathscr{C} \rightarrow X$ are appropriate functions that satisfy some assumptions. $L_{2}^{0}$ and $\mathscr{C}$ are given spaces to be defined later. $x_{0}$ is an $\mathscr{F}_{0}$-measurable random variable with finite second moment.

The aim of this paper is to study the existence of mild solutions and optimal controls for system (1). Byszewski et al. [32] verified that the nonlocal condition can be applied in physics with a better effect than the classical initial condition. For example, $g(x)$ may be given by

$$
g(x)=\sum_{i=1}^{m} c_{i} x\left(t_{i}\right)
$$


where $c_{i}(i=1, \ldots, m)$ are given constants and $0<t_{1}<\cdots<t_{m} \leq b$. The study of equations with nonlocal conditions is therefore meaningful.

An outline of this paper is given as follows. Section 2 introduces some notations and preliminary facts. In Sect. 3, the existence and uniqueness of mild solutions for system (1) are established. Optimal control results are proved in Sect. 4. Section 5 presents an example. Finally, a conclusion is given in Sect. 6.

\section{Preliminaries}

Some preliminary facts are presented in this section, which is necessary for this paper. For more details of this section, see $[1,2,5,6]$.

By $C(J, X)$ and $C\left(J^{\prime}, X\right)$ we denote the spaces of all continuous functions from $J$ to $X$ and $J^{\prime}$ to $X$, respectively. We denote

$$
\begin{aligned}
C\left(J, L^{2}(\Omega, X)\right)= & \left\{x: J \rightarrow L^{2}(\Omega, X) \mid x \text { is an } \mathscr{F}_{t}\right. \text {-adapted stochastic process, which } \\
& \text { is a continuous mapping such that } \left.\sup _{t \in J} E\|x(t)\|^{2}<\infty\right\} .
\end{aligned}
$$

It is a Banach space with the norm $\|x\|_{C\left(, L^{2}(\Omega, X)\right)}=\left(\sup _{t \in J} E\|x(t)\|^{2}\right)^{\frac{1}{2}}$.

Let $\alpha=v+\mu-v \mu$, then $1-\alpha=(1-v)(1-\mu) \geq 0$, define

$$
C_{1-\alpha}\left(J, L^{2}(\Omega, X)\right)=\left\{x \in C\left(J^{\prime}, L^{2}(\Omega, X)\right) \mid t^{1-\alpha} x(t) \in C\left(J, L^{2}(\Omega, X)\right)\right\} .
$$

For brevity, let us take $\mathscr{C}=C_{1-\alpha}\left(J, L^{2}(\Omega, X)\right)$. The space $\mathscr{C}$ equipped with the norm $\|x\|_{\mathscr{C}}=\left(\sup _{t \in J} E\left\|t^{1-\alpha} x(t)\right\|^{2}\right)^{\frac{1}{2}}$ is a Banach space.

Let $W: J \times \Omega \rightarrow K$ be a standard $Q-W i e n e r$ process on $(\Omega, \mathscr{F}, P)$ with the linear bounded covariance operator $Q$ such that $\operatorname{Tr} Q<\infty$, which is adapted to normal filtration $\left\{\mathscr{F}_{t}\right\}_{t \in[0, b]}$. Assume that there exist a complete orthonormal system $\left\{e_{n}\right\}_{n \geq 1}$ in $K$, a bounded sequence of nonnegative real numbers $\left\{\lambda_{n}\right\}_{n \in N}$ such that

$$
Q e_{n}=\lambda_{n} e_{n}, \quad \lambda_{n} \geq 0, n=1,2, \ldots
$$

and a sequence of independent real-valued Brownian motions $\left\{\beta_{n}\right\}_{n \geq 1}$ such that

$$
\langle W(t), e\rangle=\sum_{n=1}^{\infty} \sqrt{\lambda_{n}}\left\langle e_{n}, e\right\rangle \beta_{n}(t), \quad e \in K, t \in[0, b] .
$$

Introduce the Hilbert space

$$
L_{2}^{0}=\left\{f \mid f \text { is a Hilbert-Schmidt operator from } Q^{\frac{1}{2}}(K) \text { to } X\right\},
$$

whose inner product is defined by

$$
\langle\psi, \phi\rangle_{L_{2}^{0}}=\operatorname{tr}\left[\psi Q \phi^{*}\right], \quad \psi, \phi \in L_{2}^{0}
$$


Definition 1 ([1]) The fractional integral of order $q$ with the lower limit 0 for a function $f:[0, \infty) \rightarrow R$ can be written as

$$
I_{0^{+}}^{q} f(t)=\frac{1}{\Gamma(q)} \int_{0}^{t} \frac{f(s)}{(t-s)^{1-q}} d s, \quad t>0, q>0
$$

where $\Gamma(\cdot)$ is the gamma function.

Definition 2 ([1]) Riemann-Liouville's derivative of order $q$ with the lower limit 0 for a function $f:[0, \infty) \rightarrow R$ can be written as

$$
{ }^{L} D_{0^{+}}^{q} f(t)=\frac{1}{\Gamma(n-q)} \frac{d^{n}}{d t^{n}} \int_{0}^{t} \frac{f(s)}{(t-s)^{q+1-n}} d s, \quad t>0, n=[q]+1 .
$$

Definition 3 ([1]) Caputo's derivative of order $q$ with the lower limit 0 for a function $f:[0, \infty) \rightarrow R$ can be written as

$$
{ }^{C} D_{0^{+}}^{q} f(t)=D_{t}^{q}\left[f(t)-\sum_{k=0}^{n-1} \frac{t^{k}}{k !} f^{(k)}(0)\right], \quad t>0, n=[q]+1 .
$$

Furthermore, if $f^{(n)} \in C[0, \infty)$, then

$$
{ }^{C} D_{0^{+}}^{q} f(t)=\frac{1}{\Gamma(n-q)} \int_{0}^{t}(t-s)^{n-q-1} f^{(n)}(s) d s, \quad t>0, n=[q]+1 .
$$

Definition 4 ([5]) The Hilfer fractional derivative of order $v \in[0,1]$ and $\mu \in(0,1)$ with the lower limit 0 is defined as

$$
D_{0^{+}}^{v, \mu} f(t)=I_{0^{+}}^{\nu(1-\mu)} \frac{d}{d t} I_{0^{+}}^{(1-v)(1-\mu)} f(t)
$$

for functions such that the expression on the right-hand side exists.

\section{Remark 1 ([5])}

(i) For $v=0, \mu \in(0,1)$, the Hilfer fractional derivative corresponds to the classical Riemann-Liouville fractional derivative: $D_{0^{+}}^{0, \mu} f(t)=\frac{d}{d t} I_{0^{+}}^{1-\mu} f(t)={ }^{L} D_{0^{+}}^{\mu} f(t)$.

(ii) For $v=1, \mu \in(0,1)$, the Hilfer fractional derivative corresponds to the classical Caputo fractional derivative: $D_{0^{+}}^{1, \mu} f(t)=I_{0^{+}}^{1-\mu} \frac{d}{d t} f(t)={ }^{C} D_{0^{+}}^{\mu} f(t)$.

We introduce the Wright function $M_{\mu}$, which is defined by

$$
M_{\mu}(\theta)=\sum_{n=1}^{\infty} \frac{(-\theta)^{n-1}}{(n-1) \Gamma(1-n \mu)}, \quad \mu \in(0,1), \theta \in \mathbb{C},
$$

and satisfies

$$
\int_{0}^{\infty} \theta^{q} M_{\mu}(\theta) d \theta=\frac{\Gamma(1+q)}{\Gamma(1+\mu q)}, \quad \theta \geq 0 .
$$

Motivated by [6, 22], one can define the mild solution for system (1). 
Definition $5([6,22])$ A stochastic process $x \in \mathscr{C}$ is a mild solution of system (1) if $\left.I_{0^{+}}^{(1-v)(1-\mu)} x(t)\right|_{t=0}-g(x)=x_{0}$, and it satisfies the following stochastic integral equation:

$$
\begin{aligned}
x(t)= & S_{v, \mu}(t)\left[x_{0}+g(x)\right]+\int_{0}^{t} T_{\mu}(t-s) f(s, x(s)) d s \\
& +\int_{0}^{t} T_{\mu}(t-s) \sigma(s) d W(s), \quad t \in J^{\prime}
\end{aligned}
$$

where

$$
S_{v, \mu}(t)=I_{0^{+}}^{\nu(1-\mu)} T_{\mu}(t), \quad T_{\mu}(t)=t^{\mu-1} P_{\mu}(t), \quad P_{\mu}(t)=\int_{0}^{\infty} \mu \theta M_{\mu}(\theta) S\left(t^{\mu} \theta\right) d \theta .
$$

For the sake of convenience, we write (2) as

$$
\begin{aligned}
x(t)= & S_{v, \mu}(t)\left[x_{0}+g(x)\right]+\int_{0}^{t}(t-s)^{\mu-1} P_{\mu}(t-s) f(s, x(s)) d s \\
& +\int_{0}^{t}(t-s)^{\mu-1} P_{\mu}(t-s) \sigma(s) d W(s), \quad t \in J^{\prime} .
\end{aligned}
$$

Let us recall the generalized Gronwall inequality.

Lemma 1 ([33]) Assume that $\beta>0, a(t)$ is a nonnegative function locally integrable on $J$ and $b(t)$ is a nonnegative, nondecreasing continuous function defined on $J, b(t) \leq C$ ( $C$ is a constant) and suppose that $y(t)$ is nonnegative locally integrable on $J$ with

$$
y(t) \leq a(t)+b(t) \int_{0}^{t}(t-s)^{\beta-1} y(s) d s, \quad t \in J .
$$

Then

$$
y(t) \leq a(t)+\int_{0}^{t}\left[\sum_{n=1}^{\infty} \frac{[b(t) \Gamma(\beta)]^{n}}{\Gamma(n \beta)}(t-s)^{n \beta-1} a(s)\right] d s, \quad t \in J .
$$

Furthermore, if $a(t)$ is a nondecreasing function on $J$, then

$$
y(t) \leq a(t) E_{\beta}\left(b(t) \Gamma(\beta) t^{\beta}\right)
$$

where $E_{\beta}$ is the Mittag-Leffler function defined by

$$
E_{\beta}(z)=\sum_{k=0}^{\infty} \frac{z^{k}}{\Gamma(k \beta+1)}
$$

Lemma $2([34,35])$ For arbitrary $L_{2}^{0}$-valued predictable process $\Psi(t), t \in\left[\tau_{1}, \tau_{2}\right]$, which satisfies

$$
E\left(\int_{\tau_{1}}^{\tau_{2}}\|\Psi(s)\|_{L_{2}^{0}}^{2} d s\right)<\infty, \quad 0 \leq \tau_{1}<\tau_{2} \leq b
$$


we have

$$
E\left\|\int_{\tau_{1}}^{\tau_{2}} \Psi(s) d W(s)\right\|^{2} \leq E\left(\int_{\tau_{1}}^{\tau_{2}}\|\Psi(s)\|_{L_{2}^{0}}^{2} d s\right) .
$$

We introduce the following assumption.

$\left(\mathrm{H}_{0}\right) S(t)$ is continuous in the uniform operator topology for $t>0$ and $\{S(t)\}_{t \geq 0}$ is uniformly bounded, i.e., there exists $M>1$ such that $\sup _{t \in[0, \infty)}|S(t)|<M$.

Lemma 3 ([6]) Assume that $\left(\mathrm{H}_{0}\right)$ is satisfied, we have the following properties.

(i) $P_{\mu}(t), T_{\mu}(t)$, and $S_{v, \mu}(t)$ are linear and bounded operators, that is, for $\forall t \geq 0, x \in X$,

$$
\begin{aligned}
& \left\|P_{\mu}(t) x\right\| \leq \frac{M\|x\|}{\Gamma(\mu)}, \quad\left\|T_{\mu}(t) x\right\| \leq \frac{M t^{\mu-1}\|x\|}{\Gamma(\mu)} \quad \text { and } \\
& \left\|S_{v, \mu}(t) x\right\| \leq \frac{M t^{\alpha-1}\|x\|}{\Gamma(\alpha)}, \quad \alpha=v+\mu-v \mu .
\end{aligned}
$$

(ii) Operators $P_{\mu}(t), T_{\mu}(t)$, and $S_{v, \mu}(t)$ are strongly continuous.

\section{Existence of mild solutions}

The existence and uniqueness of mild solutions for system (1) are investigated in this section. Let us introduce the following hypotheses.

$\left(\mathrm{H}_{1}\right)$ : There exist a function $\psi \in L^{2}\left(J, R^{+}\right)$and a constant $c_{1}>0$ such that, for $\forall t \in J$, $\forall x \in X$

$$
\|f(t, x)\| \leq \psi(t)+c_{1} t^{1-\alpha}\|x\|
$$

$\left(\mathrm{H}_{2}\right)$ : There exists a constant $l_{1}>0$ such that, for $\forall t \in J, \forall x_{1}, x_{2} \in X$,

$$
\left\|f\left(t, x_{1}\right)-f\left(t, x_{2}\right)\right\| \leq l_{1} t^{1-\alpha}\left\|x_{1}-x_{2}\right\| .
$$

$\left(\mathrm{H}_{3}\right)$ : There exists a constant $p>\frac{1}{2 \mu-1}$ such that the function $\sigma: J \rightarrow L_{2}^{0}$ satisfies

$$
\int_{0}^{b}\|\sigma(s)\|_{L_{2}^{0}}^{2 p} d s<\infty
$$

$\left(\mathrm{H}_{4}\right)$ : There exists a constant $c_{2}>0$ such that, for $\forall x \in \mathscr{C}$,

$$
\|g(x)\| \leq c_{2}\left(1+\|x\|_{\mathscr{C}}\right)
$$

$\left(\mathrm{H}_{5}\right)$ : There exists a constant $l_{2}>0$ such that, for $\forall x_{1}, x_{2} \in \mathscr{C}$,

$$
\left\|g\left(x_{1}\right)-g\left(x_{2}\right)\right\| \leq l_{2}\left\|y x_{1}-x_{2}\right\|_{\mathscr{C}} .
$$

Theorem 1 Assume that hypotheses $\left(\mathrm{H}_{0}\right)-\left(\mathrm{H}_{5}\right)$ hold. Then system (1) has a unique mild solution on $\mathscr{C}$ provided that

$$
\frac{2 M^{2} l_{2}^{2}}{\Gamma^{2}(\alpha)}+\frac{2 M^{2} l_{1}^{2} b^{2-2 \alpha+2 \mu}}{\Gamma^{2}(\alpha)(2 \mu-1)}<1
$$


Proof Define the operator $T$ on $\mathscr{C}$ by

$$
\begin{aligned}
(T x)(t)= & S_{v, \mu}(t)\left[x_{0}+g(x)\right]+\int_{0}^{t}(t-s)^{\mu-1} P_{\mu}(t-s) f(s, x(s)) d s \\
& +\int_{0}^{t}(t-s)^{\mu-1} P_{\mu}(t-s) \sigma(s) d W(s), \quad t \in J^{\prime} .
\end{aligned}
$$

We will prove that $T$ has a fixed point on $\mathscr{C}$. The proof will be divided into two steps.

Step 1: $T$ maps $\mathscr{C}$ into $\mathscr{C}$.

For any $y \in C\left(J, L^{2}(\Omega, X)\right)$, let $x(t)=t^{\alpha-1} y(t) \in \mathscr{C}$. Define the operator $F: C\left(J, L^{2}(\Omega, X)\right) \rightarrow$ $C\left(J, L^{2}(\Omega, X)\right)$ as follows:

$$
\begin{aligned}
(F y)(t)= & t^{1-\alpha}(T x)(t) \\
= & t^{1-\alpha} S_{v, \mu}(t)\left[x_{0}+g(x)\right]+t^{1-\alpha} \int_{0}^{t}(t-s)^{\mu-1} P_{\mu}(t-s) f(s, x(s)) d s \\
& +t^{1-\alpha} \int_{0}^{t}(t-s)^{\mu-1} P_{\mu}(t-s) \sigma(s) d W(s), \quad t \in J .
\end{aligned}
$$

By Lemma 2, Lemma 3, $\left(\mathrm{H}_{1}\right)$ and $\left(\mathrm{H}_{3}\right)$, we have

$$
\begin{aligned}
& E\left\|t^{1-\alpha} \int_{0}^{t}(t-s)^{\mu-1} P_{\mu}(t-s) f(s, x(s)) d s\right\|^{2} \rightarrow 0 \quad \text { as } t \rightarrow 0^{+}, \\
& E\left\|t^{1-\alpha} \int_{0}^{t}(t-s)^{\mu-1} P_{\mu}(t-s) \sigma(s) d W(s)\right\|^{2} \rightarrow 0 \quad \text { as } t \rightarrow 0^{+},
\end{aligned}
$$

and

$$
\lim _{t \rightarrow 0^{+}}(F y)(t)=\lim _{t \rightarrow 0^{+}} t^{1-\alpha} S_{v, \mu}(t)\left[x_{0}+g(x)\right]=\frac{\left[x_{0}+g(x)\right]}{\Gamma(\alpha)} .
$$

Hence, we can define $(F y)(0)=\frac{\left[x_{0}+g(x)\right]}{\Gamma(\alpha)}$. In order to prove $T$ maps $\mathscr{C}$ into $\mathscr{C}$, we will prove that $F$ maps $C\left(J, L^{2}(\Omega, X)\right)$ into $C\left(J, L^{2}(\Omega, X)\right)$. We divide the proof into two claims.

Claim 1 For any $y(t)=t^{1-\alpha} x(t) \sup _{t \in J} E\|(F y)(t)\|^{2}<\infty$.

For any $y(t)=t^{1-\alpha} x(t) \in C\left(J, L^{2}(\Omega, X)\right)$, by Lemma 3, Hölder's inequality, $\left(\mathrm{H}_{1}\right)$ and $\left(\mathrm{H}_{3}\right)$, we have

$$
\begin{aligned}
E\|(F y)(t)\|^{2} & \\
\leq & 3 E\left\|t^{1-\alpha} S_{v, \mu}(t)\left[x_{0}+g(x)\right]\right\|^{2}+3 E\left\|t^{1-\alpha} \int_{0}^{t}(t-s)^{\mu-1} P_{\mu}(t-s) f(s, x(s)) d s\right\|^{2} \\
& +3 E\left\|t^{1-\alpha} \int_{0}^{t}(t-s)^{\mu-1} P_{\mu}(t-s) \sigma(s) d W(s)\right\|^{2} \\
\leq & \frac{3 M^{2} E\left\|x_{0}+g(x)\right\|^{2}}{\Gamma^{2}(\alpha)}+\frac{3 b^{2-2 \alpha} M^{2}}{\Gamma^{2}(\mu)} E\left(\int_{0}^{t}(t-s)^{\mu-1}\left(\psi(s)+c_{1} s^{1-\alpha}\|x(s)\|\right) d s\right)^{2} \\
& +\frac{3 b^{2-2 \alpha} M^{2}}{\Gamma^{2}(\mu)} E\left(\int_{0}^{t}(t-s)^{2 \mu-2}\|\sigma(s)\|_{L_{2}^{0}}^{2} d s\right)
\end{aligned}
$$




$$
\begin{aligned}
\leq & \frac{6 M^{2}\left[E\left\|x_{0}\right\|^{2}+2 c_{2}^{2}\left(1+\|x\|_{\mathscr{C}}^{2}\right)\right]}{\Gamma^{2}(\alpha)}+\frac{6 b^{1-2 \alpha+2 \mu} M^{2}\|\psi\|_{L^{2}\left(, R^{+}\right)}^{2}}{(2 \mu-1) \Gamma^{2}(\mu)} \\
& +\frac{6 b^{2-2 \alpha+2 \mu} M^{2} c_{1}^{2}\|x\|_{\mathscr{C}}^{2}}{\Gamma^{2}(\mu)(2 \mu-1)}+\frac{3 b^{2-2 \alpha} M^{2}}{\Gamma^{2}(\mu)}\left(\frac{p-1}{2 p \mu-p-1} b^{\frac{2 p \mu-p-1}{p-1}}\right)^{\frac{p-1}{p}} \\
& \times\left(\int_{0}^{b}\|\sigma(s)\|_{L_{2}^{0}}^{2 p} d s\right)^{\frac{1}{p}}
\end{aligned}
$$

Hence, $\sup _{t \in J} E\|(F y)(t)\|^{2}<\infty$.

Claim 2 For any $y(t)=t^{1-\alpha} x(t), t \rightarrow(F y)(t)$ is continuous on $J$ in $L^{2}(\Omega, X)$-sense.

For $t_{1}=0,0<t_{2} \leq b$, by (4), (5), and (6), we can easily get

$$
E\left\|(F y)\left(t_{2}\right)-(F y)(0)\right\|^{2} \rightarrow 0 \quad \text { as } t_{2} \rightarrow t_{1} .
$$

For $0<t_{1}<t_{2} \leq b$, we have

$$
\begin{aligned}
& E\left\|(F y)\left(t_{2}\right)-(F y)\left(t_{1}\right)\right\|^{2} \\
& \leq 3 E\left\|t_{2}^{1-\alpha} S_{v, \mu}\left(t_{2}\right)\left(x_{0}+g(x)\right)-t_{1}^{1-\alpha} S_{v, \mu}\left(t_{1}\right)\left(x_{0}+g(x)\right)\right\|^{2} \\
&+3 E \| t_{2}^{1-\alpha} \int_{0}^{t_{2}}\left(t_{2}-s\right)^{\mu-1} P_{\mu}\left(t_{2}-s\right) f(s, x(s)) d s \\
&-t_{1}^{1-\alpha} \int_{0}^{t_{1}}\left(t_{1}-s\right)^{\mu-1} P_{\mu}\left(t_{1}-s\right) f(s, x(s)) d s \|^{2} \\
&+3 E \| t_{2}^{1-\alpha} \int_{0}^{t_{2}}\left(t_{2}-s\right)^{\mu-1} P_{\mu}\left(t_{2}-s\right) \sigma(s) d W(s) \\
& \quad-t_{1}^{1-\alpha} \int_{0}^{t_{1}}\left(t_{1}-s\right)^{\mu-1} P_{\mu}\left(t_{1}-s\right) \sigma(s) d W(s) \|^{2} \\
&:= I_{1}+I_{2}+I_{3} .
\end{aligned}
$$

For $I_{1}$, we have

$$
\begin{aligned}
I_{1} \leq & 6 E\left\|t_{2}^{1-\alpha} S_{v, \mu}\left(t_{2}\right)\left(x_{0}+g(x)\right)-t_{2}^{1-\alpha} S_{v, \mu}\left(t_{1}\right)\left(x_{0}+g(x)\right)\right\|^{2} \\
& +6 E\left\|t_{2}^{1-\alpha} S_{v, \mu}\left(t_{1}\right)\left(x_{0}+g(x)\right)-t_{1}^{1-\alpha} S_{v, \mu}\left(t_{1}\right)\left(x_{0}+g(x)\right)\right\|^{2} \\
\leq & 6 t_{2}^{2-2 \alpha} E\left\|S_{v, \mu}\left(t_{2}\right)\left(x_{0}+g(x)\right)-S_{v, \mu}\left(t_{1}\right)\left(x_{0}+g(x)\right)\right\|^{2} \\
& +\frac{12\left(t_{2}^{1-\alpha}-t_{1}^{1-\alpha}\right)^{2} M^{2} t_{1}^{2 \alpha-2}\left[E\left\|x_{0}\right\|^{2}+2 c_{2}^{2}\left(1+\|x\|_{\mathscr{C}}^{2}\right)\right]}{\Gamma^{2}(\alpha)} .
\end{aligned}
$$

By Lemma 3, we obtain $\lim _{t_{2} \rightarrow t_{1}} I_{1}=0$.

For $I_{2}$, we have

$$
\begin{aligned}
I_{2} \leq & 9 E\left\|\int_{t_{1}}^{t_{2}} t_{2}^{1-\alpha}\left(t_{2}-s\right)^{\mu-1} P_{\mu}\left(t_{2}-s\right) f(s, x(s)) d s\right\|^{2} \\
& +9 E \| \int_{0}^{t_{1}} t_{2}^{1-\alpha}\left(t_{2}-s\right)^{\mu-1} P_{\mu}\left(t_{2}-s\right) f(s, x(s)) d s
\end{aligned}
$$




$$
\begin{aligned}
& -\int_{0}^{t_{1}} t_{1}^{1-\alpha}\left(t_{1}-s\right)^{\mu-1} P_{\mu}\left(t_{2}-s\right) f(s, x(s)) d s \|^{2} \\
& +9 E \| \int_{0}^{t_{1}} t_{1}^{1-\alpha}\left(t_{1}-s\right)^{\mu-1} P_{\mu}\left(t_{2}-s\right) f(s, x(s)) d s \\
& \quad-\int_{0}^{t_{1}} t_{1}^{1-\alpha}\left(t_{1}-s\right)^{\mu-1} P_{\mu}\left(t_{1}-s\right) f(s, x(s)) d s \|^{2} \\
& :=I_{21}+I_{22}+I_{23} .
\end{aligned}
$$

By Lemma 3 and Hölder's inequality, we have

$$
\begin{aligned}
I_{21} & \leq \frac{18 t_{2}^{2-2 \alpha} M^{2}}{(2 \mu-1) \Gamma^{2}(\mu)}\left[\left(t_{2}-t_{1}\right)^{2 \mu-1}\|\psi\|_{L^{2}\left(J, R^{+}\right)}+c_{1}^{2}\left(t_{2}-t_{1}\right)^{2 \mu}\|x\|_{\mathscr{C}}^{2}\right] \rightarrow 0 \text { as } t_{2} \rightarrow t_{1}, \\
I_{22} & \leq \frac{18 M^{2}\left[\|\psi\|_{L^{2}\left(J, R^{+}\right)}^{2}+c_{1}^{2} t_{1}\|x\|_{\mathscr{C}}^{2}\right]}{\Gamma^{2}(\mu)}\left(\int_{0}^{t_{1}}\left|t_{2}^{1-\alpha}\left(t_{2}-s\right)^{\mu-1}-t_{1}^{1-\alpha}\left(t_{1}-s\right)^{\mu-1}\right|^{2} d s\right) \\
& \rightarrow 0 \quad \text { as } t_{2} \rightarrow t_{1} .
\end{aligned}
$$

For $\varepsilon>0$ small enough, we have

$$
\begin{aligned}
I_{23} \leq & 18 E\left\|\int_{0}^{t_{1}-\varepsilon} t_{1}^{1-\alpha}\left(t_{1}-s\right)^{\mu-1}\left[P_{\mu}\left(t_{2}-s\right)-P_{\mu}\left(t_{1}-s\right)\right] f(s, x(s)) d s\right\|^{2} \\
& +18 E\left\|\int_{t_{1}-\varepsilon}^{t_{1}} t_{1}^{1-\alpha}\left(t_{1}-s\right)^{\mu-1}\left[P_{\mu}\left(t_{2}-s\right)-P_{\mu}\left(t_{1}-s\right)\right] f(s, x(s)) d s\right\|^{2} \\
\leq & \frac{36 t_{1}^{2-2 \alpha}\left(t_{1}^{2 \mu-1}-\varepsilon^{2 \mu-1}\right)\left[\|\psi\|_{L^{2}\left(J, R^{+}\right)}^{2}+c_{1}^{2}\left(t_{1}-\varepsilon\right)\|x\|_{\mathscr{C}}^{2}\right]}{(2 \mu-1)} \\
& \times\left(\sup _{s \in\left[0, t_{1}-\varepsilon\right]}\left\|P_{\mu}\left(t_{2}-s\right)-P_{\mu}\left(t_{1}-s\right)\right\|\right)^{2} \\
& +\frac{144 M^{2} t_{1}^{2-2 \alpha}}{\Gamma^{2}(\mu)}\left[\frac{\varepsilon^{2 \mu-1}\|\psi\|_{L^{2}\left(J, R^{+}\right)}^{2}}{2 \mu-1} \frac{c_{1}^{2} \varepsilon^{2 \mu}\|x\|_{\mathscr{C}}^{2}}{2 \mu-1}\right] \\
\rightarrow & 0 \quad \text { as } t_{2} \rightarrow t_{1}, \varepsilon \rightarrow 0 .
\end{aligned}
$$

Thus, we obtain $\lim _{t_{2} \rightarrow t_{1}} I_{2}=0$.

For $I_{3}$, we have

$$
\begin{aligned}
I_{3} \leq & 9 E\left\|\int_{t_{1}}^{t_{2}} t_{2}^{1-\alpha}\left(t_{2}-s\right)^{\mu-1} P_{\mu}\left(t_{2}-s\right) \sigma(s) d W(s)\right\|^{2} \\
& +9 E \| \int_{0}^{t_{1}} t_{2}^{1-\alpha}\left(t_{2}-s\right)^{\mu-1} P_{\mu}\left(t_{2}-s\right) \sigma(s) d W(s) \\
& -\int_{0}^{t_{1}} t_{1}^{1-\alpha}\left(t_{1}-s\right)^{\mu-1} P_{\mu}\left(t_{2}-s\right) \sigma(s) d W(s) \|^{2} \\
& +9 E \| \int_{0}^{t_{1}} t_{1}^{1-\alpha}\left(t_{1}-s\right)^{\mu-1} P_{\mu}\left(t_{2}-s\right) \sigma(s) d W(s) \\
& -\int_{0}^{t_{1}} t_{1}^{1-\alpha}\left(t_{1}-s\right)^{\mu-1} P_{\mu}\left(t_{1}-s\right) \sigma(s) d W(s) \|^{2} \\
:= & I_{31}+I_{32}+I_{33} .
\end{aligned}
$$


By Lemma 2, Lemma 3, and Hölder's inequality, we have

$$
\begin{aligned}
I_{31} \leq & \frac{9 M^{2} t_{2}^{2-2 \alpha}}{\Gamma^{2}(\mu)}\left(\int_{t_{1}}^{t_{2}}\left(t_{2}-s\right)^{2 \mu-2}\|\sigma(s)\|_{L_{2}^{0}}^{2} d s\right) \\
\leq & \frac{9 M^{2} t_{2}^{2-2 \alpha}}{\Gamma^{2}(\mu)}\left(\frac{p-1}{2 p \mu-p-1}\left(t_{2}-t_{1}\right)^{\frac{2 p \mu-p-1}{p-1}}\right)^{\frac{p-1}{p}}\left(\int_{t_{1}}^{t_{2}}\|\sigma(s)\|_{L_{2}^{0}}^{2 p} d s\right)^{\frac{1}{p}}, \\
I_{32} \leq & \frac{9 M^{2}}{\Gamma^{2}(\mu)}\left(\int_{0}^{t_{1}}\left[t_{2}^{1-\alpha}\left(t_{2}-s\right)^{\mu-1}-t_{1}^{1-\alpha}\left(t_{1}-s\right)^{\mu-1}\right]^{\frac{2 p}{p-1}} d s\right)^{\frac{p-1}{p}}\left(\int_{0}^{t_{1}}\|\sigma(s)\|_{L_{2}^{0}}^{2 p} d s\right)^{\frac{1}{p}}, \\
I_{33} \leq & 18 t_{1}^{2-2 \alpha} \sup _{s \in\left[0, t_{1}-\varepsilon\right]}\left\|P_{\mu}\left(t_{2}-s\right)-P_{\mu}\left(t_{1}-s\right)\right\|^{2}\left(\int_{0}^{t_{1}-\varepsilon}\|\sigma(s)\|_{L_{2}^{0}}^{2 p} d s\right)^{\frac{1}{p}} \\
& \times\left(\frac{p-1}{2 \mu p-p-1} t_{1}^{\frac{2 \mu p-p-1}{p-1}}-\frac{p-1}{2 \mu p-p-1} \varepsilon^{\frac{2 \mu p-p-1}{p-1}}\right)^{\frac{p-1}{p}} \\
& +\frac{72 t_{1}^{2-2 \alpha} M^{2}}{\Gamma^{2}(\mu)}\left(\frac{p-1}{2 p \mu-p-1} \varepsilon^{\frac{2 p \mu-p-1}{p-1}}\right)^{\frac{p-1}{p}}\left(\int_{t_{1}-\varepsilon}^{t_{1}}\|\sigma(s)\|_{L_{2}^{0}}^{2 p} d s\right)^{\frac{1}{p}} .
\end{aligned}
$$

By using the analogous argument performed in $I_{2}$, we can conclude that $\lim _{t_{2} \rightarrow t_{1}} I_{3}=0$. Thus, $t \rightarrow(F y)(t)$ is continuous on $J$ in $L^{2}(\Omega, X)$-sense. By Claims $1-2$, we know that $F$ maps $C\left(J, L^{2}(\Omega, X)\right)$ into $C\left(J, L^{2}(\Omega, X)\right)$, which means that $T$ maps $\mathscr{C}$ into $\mathscr{C}$.

Step 2. $T$ is a contraction.

For $\forall x_{1}, x_{2} \in \mathscr{C}$, we have

$$
\begin{aligned}
& E\left(t^{1-\alpha}\left\|\left(T x_{1}\right)(t)-\left(T x_{2}\right)(t)\right\|\right)^{2} \\
& \leq 2 E\left(t^{1-\alpha}\left\|S_{v, \mu}(t)\left(g\left(x_{1}\right)-g\left(x_{2}\right)\right)\right\|\right)^{2} \\
&+2 E\left(t^{1-\alpha}\left\|\int_{0}^{t}(t-s)^{\mu-1} P_{\mu}(t-s)\left(f\left(s, x_{1}(s)\right)-f\left(s, x_{2}(s)\right)\right) d s\right\|\right)^{2} \\
& \leq \frac{2 M^{2} l_{2}^{2}\left\|x_{1}-x_{2}\right\|_{\mathscr{C}}^{2}}{\Gamma^{2}(\alpha)}+\frac{2 M^{2} l_{1}^{2} b^{2-2 \alpha}}{\Gamma^{2}(\mu)} E\left(\int_{0}^{t}(t-s)^{\mu-1} s^{1-\alpha}\left\|x_{1}(s)-x_{2}(s)\right\| d s\right)^{2} \\
& \leq {\left[\frac{2 M^{2} l_{2}^{2}}{\Gamma^{2}(\alpha)}+\frac{2 M^{2} l_{1}^{2} b^{2-2 \alpha+2 \mu}}{\Gamma^{2}(\alpha)(2 \mu-1)}\right]\left\|x_{1}-x_{2}\right\|_{\mathscr{C}}^{2} . }
\end{aligned}
$$

Thus, $T$ is a contradiction operator on $\mathscr{C}$. According to the contraction principle, the operator $T$ has a unique fixed point $x$, which is a mild solution of system (1). This completes the proof.

\section{Existence of optimal controls}

In this section, the existence of optimal controls is investigated.

We suppose that $U$ is a separable Hilbert space. Define

$$
\begin{aligned}
L_{\mathscr{F}}^{2}(J, U)= & \left\{u: J \times \Omega \rightarrow U \mid u \text { is } \mathscr{F}_{t}\right. \text {-adapted stochastic process and } \\
& \left.E \int_{0}^{t}\|u(t)\|^{2} d t<\infty\right\}
\end{aligned}
$$


Let $V$ be a nonempty bounded closed and convex subset of $U$. Define the admissible control set

$$
U_{\mathrm{ad}}=\left\{u(\cdot) \in L_{\mathscr{F}}^{2}(J, U) \mid u(t) \in V \text { a.e. } t \in J\right\} .
$$

Consider the following controlled system:

$$
\left\{\begin{array}{l}
D_{0^{+}}^{v, \mu} x(t)=A x(t)+f(t, x(t))+B(t) u(t)+\sigma(t) \frac{d W(t)}{d t}, \quad t \in J^{\prime}, \\
\left.I_{0^{+}}^{(1-v)(1-\mu)} x(t)\right|_{t=0}-g(x)=x_{0},
\end{array}\right.
$$

where $B \in L_{\infty}(J, L(U, X))$, the control function $u \in U_{\mathrm{ad}} . L_{\infty}(J, L(U, X))$ denotes the space of operator-valued functions which are measurable in the strong operator topology and uniformly bounded on the interval $J$. We denote the norm of operator $B$ by $\|B\|_{\infty}$.

Consider the Lagrange problem $(\mathscr{P})$ : Find $\left(x^{0}, u^{0}\right) \in \mathscr{C} \times U_{\text {ad }}$ such that

$$
\mathscr{J}\left(x^{0}, u^{0}\right) \leq \mathscr{J}\left(x^{u}, u\right), \quad \forall u \in U_{\mathrm{ad}}
$$

where

$$
\mathscr{J}\left(x^{u}, u\right)=E\left\{\int_{0}^{b} \mathscr{L}\left(t, x^{u}(t), u(t)\right) d t\right\}
$$

and $x^{u}$ denotes the mild solution of system (7) corresponding to the control $u \in U_{\mathrm{ad}}$. Introduce the following assumption.

$\left(\mathrm{H}_{6}\right)$ : (i) The function $\mathscr{L}: J \times X \times U \rightarrow R \cup\{\infty\}$ is Borel measurable,

(ii) $\mathscr{L}(t, \cdot, \cdot)$ is sequentially lower semicontinuous on $X \times U$ for almost all $t \in J$,

(iii) $\mathscr{L}(t, x, \cdot)$ is convex on $U$ for $\forall x \in X$ and almost all $t \in J$,

(iv) there exist constants $d \geq 0, e>0$, and $\phi \in L^{1}\left(J, R^{+}\right)$such that

$$
\mathscr{L}(t, x, u) \geq \phi(t)+d\|x\|+e\|u\|^{2} .
$$

Theorem 2 Assume that conditions $\left(\mathrm{H}_{0}\right)-\left(\mathrm{H}_{5}\right)$ and (3) are satisfied. For $\forall u \in U_{\mathrm{ad}}$, system (7) has a unique mild solution on $\mathscr{C}$.

Proof Define the operator $\widetilde{T}$ on $\mathscr{C}$ by

$$
\begin{aligned}
(\widetilde{T} x)(t)= & S_{v, \mu}(t)\left[x_{0}+g(x)\right]+\int_{0}^{t}(t-s)^{\mu-1} P_{\mu}(t-s) f(s, x(s)) d s \\
& +\int_{0}^{t}(t-s)^{\mu-1} P_{\mu}(t-s) B(s) u(s) d s \\
& +\int_{0}^{t}(t-s)^{\mu-1} P_{\mu}(t-s) \sigma(s) d W(s), \quad t \in J^{\prime} .
\end{aligned}
$$

Compared with Theorem 1, we only need to check the term

$$
\int_{0}^{t}(t-s)^{\mu-1} P_{\mu}(t-s) B(s) u(s) d s
$$


By Lemma 3 and Hölder's inequality, we have

$$
\begin{aligned}
& E\left\|t^{1-\alpha} \int_{0}^{t}(t-s)^{\mu-1} P_{\mu}(t-s) B(s) u(s) d s\right\|^{2} \\
& \leq \frac{b^{2-2 \alpha} M^{2}\|B\|_{\infty}^{2}}{\Gamma^{2}(u)} E\left(\int_{0}^{t}(t-s)^{\mu-1}\|u(s)\| d s\right)^{2} \\
& \leq \frac{b^{2-2 \alpha} M^{2}\|B\|_{\infty}^{2}}{\Gamma^{2}(u)}\left(\int_{0}^{t}(t-s)^{2 \mu-2} d s\right) E\left(\int_{0}^{t}\|u(s)\|^{2} d s\right) \\
& \leq \frac{b^{1+2 \mu-2 \alpha} M^{2}\|B\|_{\infty}^{2}}{(2 \mu-1) \Gamma^{2}(\mu)} E\left(\int_{0}^{t}\|u(s)\|^{2} d s\right) \\
&<\infty .
\end{aligned}
$$

On the other hand, for $0<t_{1}<t_{2} \leq b$ and $\varepsilon>0$ small enough, we have

$$
\begin{aligned}
& E\left\|t_{2}^{1-\alpha} \int_{0}^{t_{2}}\left(t_{2}-s\right)^{\mu-1} P_{\mu}\left(t_{2}-s\right) B(s) u(s) d s-t_{1}^{1-\alpha} \int_{0}^{t_{1}}\left(t_{1}-s\right)^{\mu-1} P_{\mu}\left(t_{1}-s\right) B(s) u(s) d s\right\|^{2} \\
& \leq 3 E\left\|\int_{t_{1}}^{t_{2}} t_{2}^{1-\alpha}\left(t_{2}-s\right)^{\mu-1} P_{\mu}\left(t_{2}-s\right) B(s) u(s) d s\right\|^{2} \\
& +3 E \| \int_{0}^{t_{1}} t_{2}^{1-\alpha}\left(t_{2}-s\right)^{\mu-1} P_{\mu}\left(t_{2}-s\right) B(s) u(s) d s \\
& -\int_{0}^{t_{1}} t_{1}^{1-\alpha}\left(t_{1}-s\right)^{\mu-1} P_{\mu}\left(t_{2}-s\right) B(s) u(s) d s \|^{2} \\
& +3 E \| \int_{0}^{t_{1}} t_{1}^{1-\alpha}\left(t_{1}-s\right)^{\mu-1} P_{\mu}\left(t_{2}-s\right) B(s) u(s) d s \\
& -\int_{0}^{t_{1}} t_{1}^{1-\alpha}\left(t_{1}-s\right)^{\mu-1} P_{\mu}\left(t_{1}-s\right) B(s) u(s) d s \|^{2} \\
& \leq \frac{3 t_{2}^{2-2 \alpha}\left(t_{2}-t_{1}\right)^{2 \mu-1} M^{2}\|B\|_{\infty}^{2}}{(2 \mu-1) \Gamma^{2}(\mu)} E\left(\int_{t_{1}}^{t_{2}}\|u(s)\|^{2} d s\right) \\
& +\frac{3 M^{2}\|B\|_{\infty}^{2}}{\Gamma^{2}(\mu)}\left(\int_{0}^{t_{1}}\left|t_{2}^{1-\alpha}\left(t_{2}-s\right)^{\mu-1}-t_{1}^{1-\alpha}\left(t_{1}-s\right)^{\mu-1}\right|^{2} d s\right) E\left(\int_{0}^{t_{1}}\|u(s)\|^{2} d s\right) \\
& +\frac{6 t_{1}^{2-2 \alpha}\|B\|_{\infty}^{2}\left(t_{1}^{2 \mu-1}-\varepsilon^{2 \mu-1}\right)}{(2 \mu-1)} E\left(\int_{0}^{t_{1}-\varepsilon}\|u(s)\|^{2} d s\right) \\
& \times\left(\sup _{s \in\left[0, t_{1}-\varepsilon\right]}\left\|P_{\mu}\left(t_{2}-s\right)-P_{\mu}\left(t_{1}-s\right)\right\|\right)^{2} \\
& +\frac{24 M^{2}\|B\|_{\infty}^{2} t_{1}^{2-2 \alpha} \varepsilon^{2 \mu-1}}{(2 \mu-1) \Gamma^{2}(\mu)} E\left(\int_{t_{1}-\varepsilon}^{t_{1}}\|u(s)\|^{2} d s\right) \\
& \rightarrow 0 \quad \text { as } t_{2} \rightarrow t_{1}, \varepsilon \rightarrow 0
\end{aligned}
$$

Hence, $\widetilde{T}$ maps $\mathscr{C}$ into $\mathscr{C}$. Using the same method as in Theorem 1 , one can deduce that $\widetilde{T}$ has a unique fixed point on $\mathscr{C}$, and hence we omit the detailed proof here.

Now we can give the following results on the existence of optimal controls for the Lagrange problem $(\mathscr{P})$. 
Theorem 3 Suppose that conditions $\left(\mathrm{H}_{0}\right)-\left(\mathrm{H}_{6}\right)$ and (3) are fulfilled and

$$
\frac{3 M^{2} l_{2}^{2}}{\Gamma^{2}(\alpha)}+\frac{3 M^{2} l_{1}^{2} b^{2-2 \alpha+2 \mu}}{\Gamma^{2}(\alpha)(2 \mu-1)}<1
$$

then the Lagrange problem $(\mathscr{P})$ admits at least one optimal pair, that is, there exists an admissible control $u^{0} \in U_{\text {ad }}$ such that

$$
\mathscr{J}\left(x^{0}, u^{0}\right) \leq \mathscr{J}(x, u), \quad \forall u \in U_{\mathrm{ad}}
$$

Proof If $\inf \left\{\mathscr{J}\left(x^{u}, u\right) \mid u \in U_{\text {ad }}\right\}=+\infty$, there is nothing to prove. Without loss of generality, we assume that $\inf \left\{\mathscr{J}\left(x^{u}, u\right) \mid u \in U_{\mathrm{ad}}\right\}=l<+\infty$. By $\left(\mathrm{H}_{6}\right)$, we obtain $l>-\infty$. By the definition of infimum, there exists a minimizing sequence feasible pair $\left\{\left(x^{m}, u^{m}\right)\right\}$ such that

$$
\mathscr{J}\left(x^{m}, u^{m}\right) \rightarrow l \text { as } m \rightarrow+\infty
$$

where $x^{m}$ is a mild solution of system (7) corresponding to the control $u^{m} \in U_{\mathrm{ad}}$. Since $\left\{u^{m}\right\} \subseteq U_{\text {ad }}$ is a bounded subset of the reflexive Banach space $L_{\mathscr{F}}^{2}(J, U)$, there exists a subsequence, we still denote it by $\left\{u^{m}\right\}$, which weakly converges to $u^{0} \in L_{\mathscr{F}}^{2}(J, U)$. Since $U_{\text {ad }}$ is closed and convex, then by Marzur lemma $u^{0} \in U_{\mathrm{ad}}$.

Let

$$
\begin{aligned}
x^{m}(t)= & S_{v, \mu}(t)\left[x_{0}+g\left(x^{m}\right)\right]+\int_{0}^{t}(t-s)^{\mu-1} P_{\mu}(t-s) f\left(s, x^{m}(s)\right) d s \\
& +\int_{0}^{t}(t-s)^{\mu-1} P_{\mu}(t-s) B(s) u^{m}(s) d s \\
& +\int_{0}^{t}(t-s)^{\mu-1} P_{\mu}(t-s) \sigma(s) d W(s), \quad t \in J^{\prime}, \\
x^{0}(t)= & S_{v, \mu}(t)\left[x_{0}+g\left(x^{0}\right)\right]+\int_{0}^{t}(t-s)^{\mu-1} P_{\mu}(t-s) f\left(s, x^{0}(s)\right) d s \\
& +\int_{0}^{t}(t-s)^{\mu-1} P_{\mu}(t-s) B(s) u^{0}(s) d s \\
& +\int_{0}^{t}(t-s)^{\mu-1} P_{\mu}(t-s) \sigma(s) d W(s), \quad t \in J^{\prime} .
\end{aligned}
$$

By Theorem 1 and Theorem 2, one can easily get that there exists a constant $\rho>0$ such that $\left\|x^{m}\right\|_{\mathscr{C}} \leq \rho,\left\|x^{0}\right\|_{\mathscr{C}} \leq \rho$.

For $\forall t \in J$, we have

$$
\begin{aligned}
& E\left\|t^{1-\alpha} x^{m}(t)-t^{1-\alpha} x^{0}(t)\right\|^{2} \\
& \leq 3 E\left\|t^{1-\alpha} S_{v, \mu}(t) g\left(x^{m}\right)-t^{1-\alpha} S_{v, \mu}(t) g\left(x^{0}\right)\right\|^{2} \\
& \quad+3 E\left\|t^{1-\alpha} \int_{0}^{t}(t-s)^{\mu-1} P_{\mu}(t-s)\left[f\left(s, x^{m}(s)\right)-f\left(s, x^{0}(s)\right)\right] d s\right\|^{2} \\
& \quad+3 E\left\|t^{1-\alpha} \int_{0}^{t}(t-s)^{\mu-1} P_{\mu}(t-s) B(s)\left[u^{m}(s)-u^{0}(s)\right] d s\right\|^{2}
\end{aligned}
$$




$$
\begin{aligned}
\leq & \frac{3 b^{3-2 \alpha} M^{2}}{\Gamma^{2}(\mu)} E\left(\int_{0}^{t}(t-s)^{2 \mu-2}\left\|f\left(s, x^{m}(s)\right)-f\left(s, x^{0}(s)\right)\right\|^{2} d s\right) \\
& +\frac{3 b^{2-2 \alpha} M^{2}\|B\|_{\infty}^{2}}{\Gamma^{2}(\mu)}\left(\int_{0}^{t}(t-s)^{2 \mu-2} d s\right) E\left(\int_{0}^{t}\left\|u^{m}(s)-u^{0}(s)\right\|^{2} d s\right) \\
\leq & \frac{3 M^{2} l_{2}^{2}\left\|x^{m}-x^{0}\right\|_{\mathscr{C}}^{2}}{\Gamma^{2}(\alpha)}+\frac{3 b^{3-2 \alpha} M^{2} l_{1}^{2}}{\Gamma^{2}(\mu)} \int_{0}^{t}(t-s)^{2 \mu-2} E\left(s^{1-\alpha}\left\|x^{m}(s)-x^{0}(s)\right\|\right)^{2} d s \\
& +\frac{3 b^{1+2 \mu-2 \alpha} M^{2}\|B\|_{\infty}^{2}}{(2 \mu-1) \Gamma^{2}(\mu)} E\left(\int_{0}^{b}\left\|u^{m}(s)-u^{0}(s)\right\|^{2} d s\right) \\
\leq & \left.\frac{3 M^{2} l_{2}^{2}}{\Gamma^{2}(\alpha)}+\frac{3 b^{2-2 \alpha+2 \mu} M^{2} l_{1}^{2}}{\Gamma^{2}(\mu)(2 \mu-1)}\right]\left\|x^{m}-x^{0}\right\|_{\mathscr{C}}^{2} \\
& +\frac{3 b^{1+2 \mu-2 \alpha} M^{2}\|B\|_{\infty}^{2}}{(2 \mu-1) \Gamma^{2}(\mu)} E\left(\int_{0}^{b}\left\|u^{m}(s)-u^{0}(s)\right\|^{2} d s\right) .
\end{aligned}
$$

Therefore

$$
\begin{aligned}
0 & \leq\left[1-\frac{3 M^{2} l_{2}^{2}}{\Gamma^{2}(\alpha)}-\frac{3 b^{2-2 \alpha+2 \mu} M^{2} l_{1}^{2}}{\Gamma^{2}(\mu)(2 \mu-1)}\right]\left\|x^{m}-x^{0}\right\|_{\mathscr{C}}^{2} \\
& \leq \frac{3 b^{1+2 \mu-2 \alpha} M^{2}\|B\|_{\infty}^{2}}{(2 \mu-1) \Gamma^{2}(\mu)} E\left(\int_{0}^{b}\left\|u^{m}(s)-u^{0}(s)\right\|^{2} d s\right) .
\end{aligned}
$$

Furthermore,

$$
\frac{3 b^{1+2 \mu-2 \alpha} M^{2}\|B\|_{\infty}^{2}}{(2 \mu-1) \Gamma^{2}(\mu)} E\left(\int_{0}^{b}\left\|u^{m}(s)-u^{0}(s)\right\|^{2} d s\right) \rightarrow 0 \quad \text { as } m \rightarrow \infty .
$$

By (8), we have $x^{m} \rightarrow x^{0}$ in $\mathscr{C}$ as $m \rightarrow \infty$. Note that $\left(\mathrm{H}_{6}\right)$ implies that the assumptions of Balder [36] are satisfied. By Balder's theorem, we have

$$
\begin{aligned}
l & =\lim _{m \rightarrow \infty} E\left\{\int_{0}^{b} \mathscr{L}\left(t, x^{m}(t), u^{m}(t)\right) d t\right\} \\
& \geq \lim _{m \rightarrow \infty} E\left\{\int_{0}^{b} \mathscr{L}\left(t, x^{0}(t), u^{0}(t)\right) d t\right\}=\mathscr{J}\left(x^{0}, u^{0}\right) \geq l .
\end{aligned}
$$

This means that $\mathscr{J}$ attains its minimum at $\left(x^{0}, u^{0}\right)$. This completes the proof.

\section{An example}

Consider the following fractional control system:

$$
\left\{\begin{aligned}
& D_{0^{+}}^{v, \frac{3}{4}} x(t, \xi)= \frac{\partial^{2}}{\partial \xi^{2}} x(t, \xi)+f(t, x(t, \xi))+2 u(t, \xi) \\
&+\sigma(t, \xi) \frac{d \beta(t)}{d t}, \quad t \in(0,1], \xi \in[0, \pi], \\
& x(t, 0)=x(t, \pi)=0, \quad t \in(0,1], \\
&\left.I_{0^{+}}^{\frac{1}{4}(1-v)} x(t, \xi)\right|_{t=0}-\sum_{i=0}^{m} \int_{0}^{\pi} \gamma(\xi, \tau) x\left(t_{i}, \tau\right) d \tau=x_{0}(\xi), \quad \xi \in[0, \pi],
\end{aligned}\right.
$$

with the cost function

$$
\mathscr{J}\left(x^{u}, u\right)=E\left\{\int_{0}^{1} \int_{0}^{\pi}\left|x^{u}(t, \xi)\right|^{2} d \xi d t+\int_{0}^{1} \int_{0}^{\pi}|u(t, \xi)|^{2} d \xi d t\right\}
$$


where $D_{0^{+}}^{v, \frac{3}{4}}$ denotes the Hilfer fractional derivative, $v \in[0,1], c>0$ is a constant. $0<t_{0}<$ $t_{1}<\cdots<t_{m} \leq 1, \gamma(\xi, \tau) \in L^{2}\left([0, \pi] \times[0, \pi], R^{+}\right) . \beta(t)$ is a one-dimensional standard Brownian motion defined on the filtered probability space $(\Omega, \mathscr{F}, P)$.

Let $X=U=L^{2}([0, \pi], R), J^{\prime}=(0,1], J=[0,1], \alpha=\frac{1}{4} v+\frac{3}{4}$. Define the operator $A: D(A) \subset$ $X \rightarrow X$ by $A \varsigma=\frac{\partial^{2} \varsigma}{\partial \xi^{2}}$, where

$$
D(A)=\left\{\varsigma \in X: \varsigma, \frac{\partial \varsigma}{\partial \xi} \text { are absolutely continuous, } \frac{\partial^{2} \varsigma}{\partial \xi^{2}} \in X, \varsigma(0)=\varsigma(\pi)=0\right\} .
$$

It is easy to check that $A$ generates a strongly continuous semigroup $\{S(t)\}_{t \geq 0}$ which is compact, analytic, and self-adjoint [4]. Hence, $\left(\mathrm{H}_{0}\right)$ is fulfilled.

Clearly, we can rewrite system (9) into the abstract form:

$$
\left\{\begin{array}{l}
D_{0^{+}}^{\nu, \frac{3}{4}} x(t)=A x(t)+f(t, x(t))+B(t) u(t)+\sigma(t) \frac{d W(t)}{d t}, \quad t \in J^{\prime}:=(0,1], \\
\left.I_{0^{+}}^{\frac{1}{4}(1-\nu)} x(t)\right|_{t=0}-g(x)=x_{0},
\end{array}\right.
$$

where

$$
\begin{aligned}
& x(t)(\xi)=x(t, \xi), \quad f(t, x(t))(\xi)=f(t, x(t, \xi)), \quad B(t) u(t)(\xi)=2 u(t, \xi), \\
& \sigma(t)(\xi)=\sigma(t, \xi), \quad g(x)(\xi)=\sum_{i=0}^{m} G x\left(t_{i}\right)(\xi), \\
& G h(\xi)=\int_{0}^{\pi} \gamma(\xi, \tau) h(\tau) d \tau, \quad \text { for } h \in X, \xi \in[0, \pi],
\end{aligned}
$$

and

$$
\mathscr{J}\left(x^{u}, u\right)=E \int_{0}^{1}\left(\left\|x^{u}(t)\right\|^{2}+\|u(t)\|^{2}\right) d t
$$

Obviously, $\left(\mathrm{H}_{6}\right)$ is satisfied. We can choose $c_{2}=l_{2}=(m+1)\left(\int_{0}^{\pi} \int_{0}^{\pi} \gamma^{2}(\xi, \tau) d \tau d \xi\right)^{\frac{1}{2}}$. Then $\left(\mathrm{H}_{4}\right)$ and $\left(\mathrm{H}_{5}\right)$ are satisfied.

Define $f(t, y(t, \xi))=\frac{t^{1-\alpha} e^{-t}|y(t, \xi)|}{\left(1+e^{t}\right)(1+|y(t, \xi)|)}$. Note that $\|f(t, y(t, \xi))\| \leq e^{-t}$. Moreover,

$$
\begin{aligned}
\left\|f\left(t, y_{1}(t)\right)(\xi)-f\left(t, y_{2}(t)\right)(\xi)\right\| & =\frac{t^{1-\alpha} e^{-t}|| y_{1}(t, \xi)|-| y_{2}(t, \xi)||}{\left(1+e^{t}\right)\left(1+\left|y_{1}(t, \xi)\right|\right)\left(1+\left|y_{2}(t, \xi)\right|\right)} \\
& \leq \frac{t^{1-\alpha} e^{-t}}{1+e^{t}}\left|y_{1}(t, \xi)-y_{2}(t, \xi)\right| \\
& \leq \frac{1}{2} t^{1-\alpha}\left|y_{1}(t, \xi)-y_{2}(t, \xi)\right| .
\end{aligned}
$$

Hence, $\left(\mathrm{H}_{1}\right)$ and $\left(\mathrm{H}_{2}\right)$ are satisfied.

If conditions (3), (8), and $\left(\mathrm{H}_{3}\right)$ are satisfied, then by Theorem 3, system (10) is approximately controllable.

\section{Conclusion}

In this paper, we study a class of Hilfer fractional stochastic differential equations. By means of stochastic analysis theory, fractional calculations, and operator semigroup the- 
ory, we obtain the existence and uniqueness of mild solutions for these equations. Moreover, the existence of optimal pairs for the corresponding Lagrange control systems is investigated. Our future work will be focused on investigating the optimal control problem of Hilfer fractional stochastic differential equations with Lévy noise.

\section{Acknowledgements}

The authors would like to thank the referees and the editor for their valuable comments which led to improvement of this work.

\section{Funding}

This work was supported by the National Natural Science Foundation of China under grant 61671002.

\section{Competing interests}

The authors declare to have no competing interests.

\section{Authors' contributions}

All authors participated in drafting and checking the manuscript and approved the final manuscript.

\section{Publisher's Note}

Springer Nature remains neutral with regard to jurisdictional claims in published maps and institutional affiliations.

Received: 5 July 2018 Accepted: 9 January 2019 Published online: 18 January 2019

\section{References}

1. Kilbas, A.A., Srivastava, H.M., Trujillo, J.J.: Theory and Applications of Fractional Differential Equations. Elsevier, New York (2006)

2. Podlubny, I.: Fractional Differential Equations: An Introduction to Fractional Derivatives, Fractional Differential Equations, to Methods of Their Solution and Some of Their Applications, vol. 198. Academic Press, San Diego (1998)

3. Zhou, Y., Jiao, F.: Existence of mild solutions for fractional neutral evolution equations. Comput. Math. Appl. 59, 1063-1077 (2010)

4. Zhou, Y., Zhang, L., Shen, X.H.: Existence of mild solutions for fractional evolution equations. J. Integral Equ. Appl. 25 557-586 (2013)

5. Hilfer, R.: Applications of Fractional Calculus in Physics. World Scientific, Singapore (2000)

6. Gu, H., Trujillo, J.J.: Existence of mild solution for evolution equation with Hilfer fractional derivative. Appl. Math. Comput. 257, 344-354 (2015)

7. Zhou, Y.: Basic Theory of Fractional Differential Equations. World Scientific, Singapore (2014)

8. Zhou, Y., Jiao, F.: Nonlocal Cauchy problem for fractional evolution equations. Nonlinear Anal., Real World Appl. 11(5), 4465-4475 (2010)

9. Li, K., Peng, J., Jia, J.: Cauchy problems for fractional differential equations with Riemann-Liouville fractional derivatives. J. Funct. Anal. 263, 476-510 (2012)

10. Wang, J.R., Fečkan, M., Zhou, Y.: On the new concept solutions and existence results for impulsive fractional evolution equations. Dyn. Partial Differ. Equ. 8(4), 345-361 (2011)

11. Gou, H., Li, B.: Study on Sobolev type Hilfer fractional integro-differential equations with delay. J. Fixed Point Theory Appl. 20(1), 44 (2018)

12. Gou, H., Li, B.: Study a class of nonlinear fractional non-autonomous evolution equations with delay. J. Pseudo-Differ Oper. Appl. 2, 1-22 (2017)

13. Wang, J.R., Ibrahim, A.G., O'Regan, D.: Topological structure of the solution set for fractional non-instantaneous impulsive evolution inclusions. J. Fixed Point Theory Appl. 20(2), 59 (2018)

14. Li, M., Wang, J.R.: Exploring delayed Mittag-Leffler type matrix functions to study finite time stability of fractional delay differential equations. Appl. Math. Comput. 324, 254-265 (2018)

15. Li, X., Yong, J.: Optimal Control Theory for Infinite Dimensional Systems. Birkhäuser, Boston (1995)

16. Wang, J.R., Zhou, Y.: A class of fractional evolution equations and optimal controls. Nonlinear Anal., Real World Appl. 12(1), 262-272 (2011)

17. Guo, T.L.: The necessary conditions of fractional optimal control in the sense of Caputo. J. Optim. Theory Appl. 156, 115-126 (2013)

18. Kumar, S.: Mild solution and fractional optimal control of semilinear system with fixed delay. J. Optim. Theory Appl. 174, 108-121 (2017)

19. Zhu, S., Fan, Z., Li, G.: Optimal controls for Riemann-Liouville fractional evolution systems without Lipschitz assumption. J. Optim. Theory Appl. 174, 47-64 (2017)

20. Wang, J.R.: Approximate mild solutions of fractional stochastic evolution equations in Hilbert spaces. Appl. Math. Comput. 256, 315-323 (2015)

21. Li, K.: Stochastic delay fractional evolution equations driven by fractional Brownian motion. Math. Methods Appl. Sci. 38(8), 1582-1591 (2015)

22. Ahmed, H.M., El-Borai, M.M.: Hilfer fractional stochastic integro-differential equations. Appl. Math. Comput. 331, 182-189 (2018)

23. Yan, Z., Jia, X.: Optimal controls for fractional stochastic functional differential equations of order $\alpha \in(1,2]$. Bull. Malays. Math. Sci. Soc. 41, 1581-1606 (2018) 
24. Balasubramaniam, P., Tamilalagan, P.: The solvability and optimal controls for impulsive fractional stochastic integro-differential equations via resolvent operators. J. Optim. Theory Appl. 174, 139-155 (2017)

25. Rihan, F.A., Rajivganthi, C., Muthukumar, P.: Fractional stochastic differential equations with Hilfer fractional derivative: Poisson jumps and optimal control. Discrete Dyn. Nat. Soc. 2017, Article ID 5394528 (2017)

26. Ahmed, H.M., Wang, J.R.: Exact null controllability of Sobolev-type Hilfer fractional stochastic differential equations with fractional Brownian motion and Poisson jumps. J. Nonlinear Sci. Appl. 44(3), 673-690 (2018)

27. Tamilalagan, P., Balasubramaniam, P.: The solvability and optimal controls for fractional stochastic differential equations driven by Poisson jumps via resolvent operators. Appl. Math. Optim. 77, 443-462 (2018)

28. Rajivganthi, C., Muthukumar, P.: Almost automorphic solutions for fractional stochastic differential equations and its optimal control. Optim. Control Appl. Methods 37, 663-681 (2016)

29. Yan, Z., Lu, F.: Existence of an optimal control for fractional stochastic partial neutral integro-differential equations with infinite delay. J. Nonlinear Sci. Appl. 8, 557-577 (2015)

30. Muthukumar, P., Thiagu, K.: Existence of solutions and approximate controllability of fractional nonlocal stochastic differential equations of order $1<q \leq 2$ with infinite delay and Poisson jumps. Differ. Equ. Dyn. Syst. 26, 15-36 (2018)

31. Nagarajan, D., Palanisamy, M.: Optimal control on semilinear retarded stochastic functional differential equations driven by Poisson jumps in Hilbert space. Bull. Korean Math. Soc. 55(2), 479-497 (2018)

32. Byszewski, L., Lakshmikantham, V:: Theorem about the existence and uniqueness of a solution of a nonlocal abstract Cauchy problem in a Banach space. Appl. Anal. 40, 11-19 (1991)

33. Ye, H.P., Gao, J.M., Ding, Y.S.: A generalized Gronwall inequality and its application to a fractional differential equation. J. Math. Anal. Appl. 328, 1075-1081 (2007)

34. Kruse, R., Larsson, S.: Optimal regularity for semilinear stochastic partial differential equations with multiplicative noise. Electron. J. Probab. 17, paper no. 65 (2012)

35. Kruse, R.: Strong and Weak Approximation of Semilinear Stochastic Evolution Equations. Lecture Notes in Mathematics. Springer, Cham (2014)

36. Balder, E.J.: Necessary and sufficient conditions for $l_{1}$-strong weak lower semicontinuity of integral functionals. Nonlinear Anal. 11, 1399-1404 (1987)

\section{Submit your manuscript to a SpringerOpen ${ }^{\circ}$ journal and benefit from:}

- Convenient online submission

- Rigorous peer review

- Open access: articles freely available online

- High visibility within the field

- Retaining the copyright to your article

Submit your next manuscript at $\boldsymbol{\nabla}$ springeropen.com 